\title{
Abrupt formation of a right atrium thrombus detected by transesophageal echocardiography during laparoscopic assisted vaginal hysterectomy and spontaneous resolution during thromboembolectomy
} -A case report-

\author{
Kwangrae Cho ${ }^{1}$, Byung-Kwan Chu ${ }^{1}$, Ilyong Han ${ }^{2}$, Chee-Mahn Shin ${ }^{1}$, Young-Jae Kim ${ }^{1}$, Soon Ho Cheong ${ }^{1,3}$, \\ Kun Moo Lee ${ }^{1}$, Se Hun Lim ${ }^{1}$, Jeong Han Lee ${ }^{1}$, Myoung-Hun Kim ${ }^{1}$, and Hyo-Joong Kim ${ }^{1}$ \\ Departments of ${ }^{1}$ Anesthesiology and Pain Medicine, ${ }^{2}$ Thoracic and Cardiovascular Surgery, Busan Paik Hospital, College of Medicine, \\ Inje University, ${ }^{3}$ Department of Paik Institute for Clinical Research, Inje University, Busan, Korea
}

Intraoperative formation and management of a thrombus in right atrium has been reported occasionally. Nevertheless, it is rare that a right atrial thrombus with unstable hemodynamic changes detected by transesophageal echocardiography is resolved spontaneously. We report upon the 44-year-old woman, who had a right atrial thrombus detected by transesophageal echocardiography during laparoscopic assisted vaginal hysterectomy and resolved during thromboembolectomy. (Korean J Anesthesiol 2012; 62: 382-386)

Key Words: Laparoscopic surgery, Right atrium, Thrombus, Transesophageal echocardiography.

Intraoperative right atrium thrombus is sometimes found together with pulmonary thrombosis or during echocardiography by chance but rarely. Since right atrium thrombus may have fatal results in pulmonary thrombosis and death, the hemodynamic changes caused by right atrium thrombus should be promptly detected, definitely diagnosed by transesophageal echocardiography, and rapidly treated thus affecting the prognosis. We report a case of a patient in which abrupt intraoperative hemodynamic changes took place and right atrium thrombus was verified by transesophageal echocardiography, but it disappeared spontaneously during thromboembolectomy along with a relevant literature review.

\section{Case Report}

A 44-year-old female patient, $155 \mathrm{~cm}$ in height and $65 \mathrm{~kg}$ in weight, who visited our hospital with a main complaint of dysmenorrhea and hypermenorrhea was diagnosed with

Received: October 25, 2010. Revised: February 18, 2011. Accepted: May 18, 2011.

Corresponding author: Kwangrae Cho, M.D., Department of Anesthesiology and Pain Medicine, Busan Paik Hospital, Gaegeum 2-dong, Busanjin-gu, Busan 614-735, Korea. Tel: 82-51-890-6520, Fax: 82-51-898-4216, E-mail: maeteo@hanmir.com

(c) This is an open-access article distributed under the terms of the Creative Commons Attribution Non-Commercial License (http:// creativecommons.org/licenses/by-nc/3.0/), which permits unrestricted non-commercial use, distribution, and reproduction in any medium, provided the original work is properly cited. 
uterine myoma and decided to undergo a laparoscopic assisted vaginal hysterectomy. The patient's past medical history showed that she had a cystectomy for an ovarian tumor ten years ago and subtotal-thyroidectomy for thyroid cancer five years ago. The patient was taking $0.15 \mathrm{mg}$ of Synthroid once a day. Since the preoperative hemoglobin levels were $7.8 \mathrm{~g} / \mathrm{dl}$, two units of packed red blood cells were transfused. There was no other specific finding in the preoperative examination.

For preanesthetic medication, an intramuscular injection of $0.2 \mathrm{mg}$ of glycopyrrolate was given. Electrocardiography, noninvasive blood pressure monitoring, pulse oxymetry, and Bispectral index (BIS) monitoring (A-3000 EEG BIS monitor, Aspect Medical system, USA) started immediately after the patient arrived at the operation room. The vital signs before the induction of anesthesia were as follows; a blood pressure of $139 / 87 \mathrm{mmHg}$, a heart rate of $70 / \mathrm{min}$, and a pulse oxygen saturation of $99 \%$. For the induction of anesthesia, $120 \mathrm{mg}$ of propofol and $40 \mathrm{mg}$ of lidocaine were mixed and intravenously injected. Then, $100 \mathrm{mg}$ of succinylcholine was intravenously injected for endotracheal intubation. During the endotracheal intubation, $7 \mathrm{~mm}$ of a single endotracheal tube was fixed with the point marked with " $21 \mathrm{~cm}$ " attached to the lips after checking that breathing sound was well heard on both sides. Vecuronium $4 \mathrm{mg}$ was intravenously injected to maintain muscle relaxation, and $2-3 \mathrm{vol} \%$ of sevoflurane, $2 \mathrm{~L} / \mathrm{min}$ of oxygen, and $2 \mathrm{~L} / \mathrm{min}$ of nitrous oxide were used to maintain the anesthesia. The tidal volume was regulated to keep the end-tidal $\mathrm{CO}_{2}$ pressure at $30-35 \mathrm{mmHg}$, and the depth of the anesthesia was controlled to maintain a BIS index of 40-60. The laparoscopic assisted vaginal hysterectomy began with the patient in the lithotomy position. The operation continued without any problems, and the vital signs of the patient were stably maintained. The intra-abdominal pressure was kept at 10-15 mmHg. Suddenly, at two hours after the initiation of the operation, the blood pressure dropped from 110/63 $\mathrm{mmHg}$ to
$78 / 41 \mathrm{mmHg}$, end-tidal $\mathrm{CO}_{2}$ pressure dropped from $45 \mathrm{mmHg}$ to $20 \mathrm{mmHg}$, and the pulse oxygen saturation dropped to $70 \%$. The peak airway pressure increased from $23 \mathrm{cmH}_{2} \mathrm{O}$ to $33 \mathrm{cmH}_{2} \mathrm{O}$, and the heart rate increased from $70 / \mathrm{min}$ to 125 / min. Promptly, $5 \mathrm{mg}$ of ephedrine and $100 \mu \mathrm{g}$ of phenylephrine were intravenously injected two times at five-minute intervals. Intravenous injection of $10 \mathrm{mg}$ of ephedrine was then done, but the blood pressure did not increase. After $100 \mu \mathrm{g}$ of epinephrine was injected, the blood pressure increased to $182 / 117 \mathrm{mmHg}$, the heart rate increased to $140 / \mathrm{min}$, and the pulse oxygen saturation was maintained at $100 \%$. Following the intravenous injection of $15 \mathrm{mg}$ of esmolol, the patient remained in a stable state with the blood pressure at 107/74 $\mathrm{mmHg}$, heart rate at 103/ min, pulse oxygen saturation at $100 \%$, end-tidal $\mathrm{CO}_{2}$ pressure at $35 \mathrm{mmHg}$, and peak airway pressure at $20 \mathrm{cmH}_{2} \mathrm{O}$. For continuous arterial pressure monitoring, a 22-gauge catheter was inserted into the radial artery in the left arm and invasive blood pressure monitoring was started. The results of the arterial blood gas analysis done at that time were a pH of 7.412, a $\mathrm{PaCO}_{2}$ of $38.9 \mathrm{mmHg}$, a $\mathrm{PO}_{2}$ of $173 \mathrm{mmHg}$, and oxygen saturation at $99.8 \%\left(\mathrm{FiO}_{2}: 0.5\right)$. Massive carbon dioxide embolism, pulmonary thrombus, tension pneumothorax, and right atrial thrombus were suspected as the cause, and a transesophageal echocardiography (HDI3000, PHILIPS, Netherlands) was done to differentiate the cause and evaluate cardiac function. A lesion that looked like a lump was found in the right atrium, and the size shown on the monitor was $2.8 \times 4.1 \mathrm{~cm}$. The shape of the lesion was round. A freely-moving echo-density was found in the region that did not overlap the right atrium, although the overall motion was not great (Fig. 1). The right atrium and the right ventricle were not hypertrophied but the size of the right atrium was larger than normal. The interventricular septum was moved toward the right ventricle in the systole. The motion of the tricuspid valve was normal, but the tricuspid regurgitation or pulmonary artery pressure could not be measured because
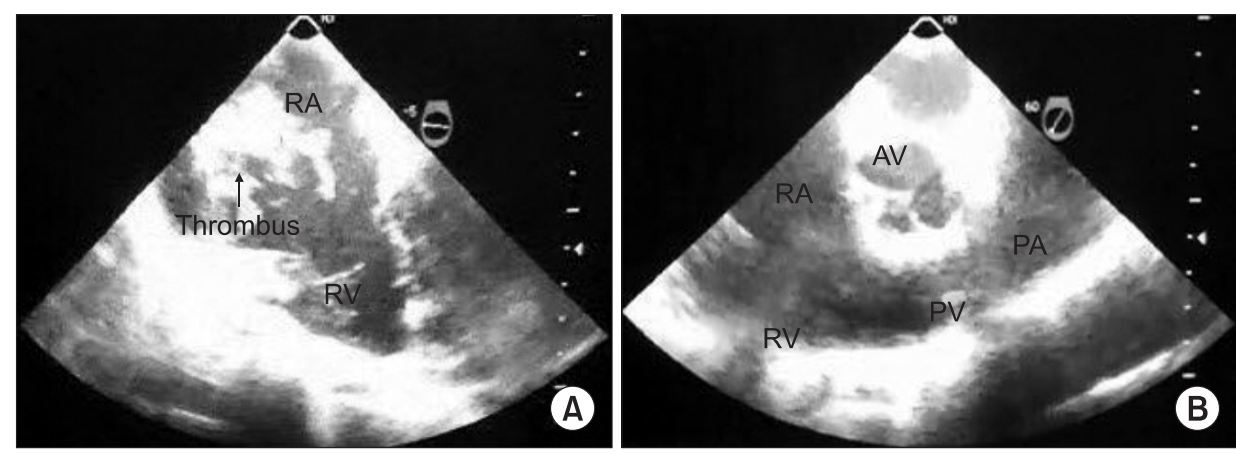

Fig. 1. (A) Transesophageal echocardiography shows a partially mobile hyperechoic thrombus in the right atrium (RA) and marked dilatation of RA, as seen on the midesophageal four-chamber view. (B) Transesophageal echocardiography shows no visible thrombus in pulmonary artery (PA), as seen on the midesophageal right ventricular inflow-outflow. These images are captured screen of transesophageal echocardiography using portable video recording devices. AV: aortic valve, PV: pulmonary valve, RV: right ventricle. 
of the lump density. The function of the left heart was normal, and thrombosis in the aorta was not suspected. We assumed that the partial movement of the thrombus to the pulmonary artery might have caused the pulmonary thrombosis because the initial vital signs had not been good even though the vital signs were normal since the thrombus was localized to the right atrium. Confirming that it was right atrium thrombosis with a cooperative diagnosis from a thoracic surgeon and a cardiology specialist, we decided to perform a thromboembolectomy, which is the most rapid treatment, and received consent from the caregiver after providing an explanation on the patient's condition since we assumed that the same phenomena could occur once again even though the vital signs were normal at that time. Although thromboembolectomy had to be rapidly performed for the treatment of right atrium thrombosis, we decided to perform it after a simple hemostasis in the obstetrics and gynecology department because the vital signs were stable at that time. No specific change was found in the right atrium thrombus during the hemostasis.

The hemostasis was finished after about 20 minutes, and a central venous catheter was inserted through the right internal jugular vein for the thromboembolectomy. The method for anesthetic maintenance was changed to intravenous injections of $10 \mu \mathrm{g} / \mathrm{kg} / \mathrm{h}$ of fentanyl, $50 \mu \mathrm{g} / \mathrm{kg} / \mathrm{h}$ of midazolam, and $0.2 \mathrm{mg} / \mathrm{kg} /$ $\mathrm{h}$ of vecuronium. When preparing for the thromboembolectomy, there was no change in the electrocardiography, capnography, end-tidal $\mathrm{CO}_{2}$ concentration, and airway pressure, and no hemodynamic change was found in the blood pressure, heart rate, and pulse oxygen saturation. After sternotomy was performed in the operation, we observed all the other structures in the heart using transesophageal echocardiography, but the

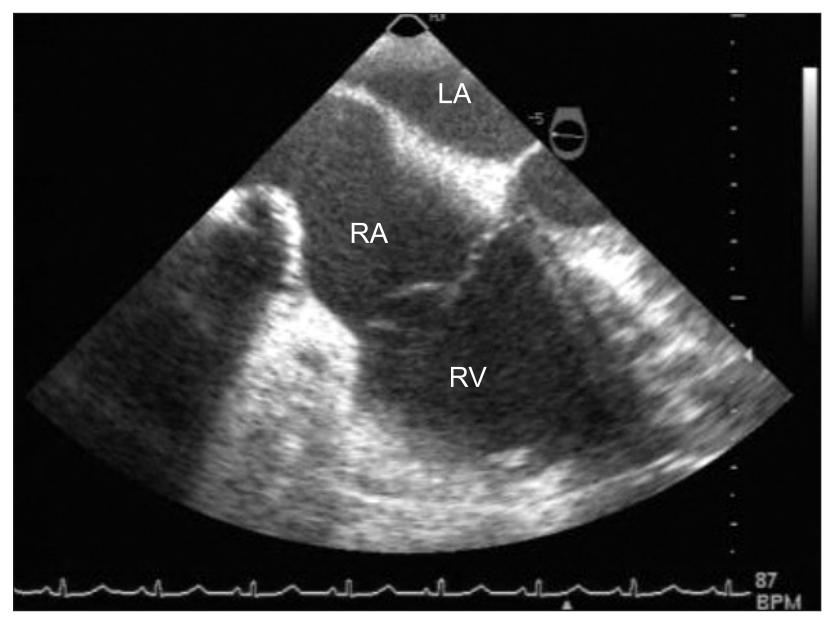

Fig. 2. Transesophageal echocardiography shows spontaneous resolution of thrombus in the right atrium (RA), as seen on the midesophageal four-chamber view. LA: left atrium, RV: right ventricle. thrombus that had existed in the right atrium was not found. Even though there was no symptom or sign that suggested movement of the thrombus to the pulmonary artery, we were not able to judge that the thrombus was spontaneously resolved. Since the possible movement of the thrombus to the pulmonary artery or other structures could not be explicitly excluded, we decided to continue the operation after consulting with the thoracic surgeon and the cardiology specialist. Aortic cannulation, superior vena cava cannulation, and inferior vena cava cannulation were smoothly performed. Following the aortic cross clamp, cardiac arrest was carried out using a cardioplegia, and open-heart surgery was done under cardiopulmonary bypass. During the cardiopulmonary bypass, the activated clotting time (ACT) was maintained for more than 480 seconds. Since the thrombus was found neither in the right atrium nor at the origin of the pulmonary artery, the catheter was deeply inserted into the right pulmonary artery and a few thrombi, $7 \mathrm{~mm}$ long and $2 \mathrm{~mm}$ thick, were eliminated by suction. The thrombi found during the operation were much smaller than the thrombus found by the transesophageal echocardiography before, but the right atrium was sutured because no more thrombus was found and there were no abnormal findings in the other regions. Cardiac function was spontaneously recovered without defibrillation, and the ACT was reversed to 145 seconds before weaning from the cardiopulmonary bypass. Transesophageal echocardiography was performed to verify cardiac function recovery and the existence of a thrombus and no specific problems were found (Fig. 2). However, another operation was performed for the purpose of hemostasis just after the thromboembolectomy since the bleeding was severe at the region where the operation was performed in the obstetrics and gynecology department. During the entire operation, 16 units of packed RBCs, 8 units of fresh frozen plasma, and 8 units of platelets were transfused.

After the operation in the obstetrics and gynecology department, the patient was transferred to the intensive care unit without reversing muscle relaxation. Extubation was performed after the patient's consciousness and muscle strength recovered on the first day after the operation. The D-dimer level increased to $8.24 \mu \mathrm{g} / \mathrm{ml}$, but lung perfusion scanning did not show any pulmonary thrombus. The patient was transferred to the general ward on the second day. The lower extremity vein Doppler scanning performed on the 14th day did not show any deep vein thrombi. The postoperative transthoracic echocardiography showed normal cardiac functions. The patient was discharged on the 30th day after the operation without any problems.

\section{Discussion}

Right heart thromboemboli represent embolized deep 
venous thromboses that are temporarily lodged in the right atrium or ventricle and are often referred to as "emboli in transit" [1]. Right atrial thrombus is defined as a right atrial mass meeting one or more of the following criteria: serpiginous mobile mass representing a cast of a leg vein; location in the right atrial appendage or on a pacing wire with no clinical evidence to suggest endocarditis; resolution with anticoagulation; or pathological confirmation during surgery. Masses with characteristic appearance of normal variant structures (Chiari network or prominent Eustachian valve) were excluded. Valvular masses (vegetations or tumors), masses attached by a stalk to the interatrial septum (myxomas), and masses subsequently proven pathologically to be tumors were also excluded [2].

The risk factors of venous thrombosis were suggested by the European Society of Cardiology (ESC): strong predisposing factors were hip or leg fracture, hip or knee joint replacement, major general operations, major injury, and spinal cord injury; moderate predisposing factors were arthroscopic knee surgery, central venous line, chemotherapy, chronic cardiac or respiratory disease, malignant cancer, and oral contraceptive therapy; and weak predisposing factors were bedrest for more than three days, old age, laparoscopic operation, obesity, varicose veins, and pregnancy [3]. The patient in this case study had only two weak risk factors, which were obesity and laparoscopic surgery. The results of the preoperative coagulation test and the electrocardiography were within normal limits. Thus, the patient had a moderate or weak risk for venous thrombosis.

We confronted the situation where intraoperative pulmonary thromboembolism was suspected and found a lump $2.8 \times 4.1 \mathrm{~cm}$ in size that was suspected as a thrombus by performing transesophageal echocardiography for the diagnosis. Schwartzbard et al. [2] found 20 cases of right atrium thrombus in a retrospective study of more than 60,000 cases of echocardiography. All 20 cases were diagnosed by transesophageal echocardiography, but when the diagnosis was made by transthoracic echocardiography, six cases were definitely diagnosed as thrombosis (30\%) and two cases were suspected as thrombosis (10\%), indicating a $60 \%$ false negative diagnosis rate among the patients. They also reported that the thrombus was found only by transesophageal echocardiography in eight (62\%) out of 13 patients who had underwent anticoagulant therapy and in three out of seven patients who had underwent the operation. Obeid et al. [4] reported that the sensitivity of transthoracic echocardiography ranged from $50 \%$ to $60 \%$ in discovering right atrium thrombus when compared with that of transesophageal echocardiography. Pruszczyk et al. [5] reported that pulmonary embolism was found in patients in whom a high degree of pulmonary embolism was suspected by transesophageal echocardiography with $80 \%$ sensitivity and $100 \%$ specificity. Hence, whenever right atrium thrombosis is suspected, transesophageal echocardiography should be done since it has an important role in the diagnosis as well as in the treatment.

Two types of right atrium thrombus are described depending on the shape, pathophysiology, and prognosis. Type A is long, thin, and freely-moving, and described as having a worm-like or snake-like shape. It exhibits a high prevalence for deep vein thrombosis and is associated with pulmonary thrombosis in almost all the cases. It is rarely caused by an anomaly in the heart itself that potentially generates the thrombus. Type B has less or no movement and the shape is nonspecific and similar to that of the thrombus in the left side of the heart. It is often caused by an anomaly of the heart itself that is in stasis. Most of the thrombi are localized to the right heart. Type B is not usually related to deep vein thrombosis. Often, it is associated with pulmonary thrombosis but not fatal. A very poor prognosis has been reported in connection with a high rate of pulmonary embolism and death in type A thrombus $[6,7]$. The thrombus found in our case was thought to be type B thrombus since the motion around the right atrium was not great and the lump shape was nonspecific. It caused unstable hemodynamic changes and was spontaneously resolved despite the large size. We were not able to accurately observe the process in which the thrombus disappeared. It was assumed that the thrombus might have been spontaneously resolved because of the delay time before the open-heart surgery, the motion needed for preparing patient for the operation, and the anticoagulation effect of the heparin that was injected before cardiopulmonary bypass procedure. Rose et al. [1] reported that 16 out of 177 patients who were confirmed to have right heart thrombus did not undergo any treatment. The most possible explanation is that the peripheral coagulation was temporarily stopped in the right atrium and then broken and put into the pulmonary circulation [8].

It was reported that the occurrence rate of right heart thrombosis ranged from $3 \%$ to $23 \%$ among patients in whom pulmonary thromboembolism was suspected $[9,10]$. Chartier et al. [9] reported that mortality was $45 \%$ among patients who had right heart thrombosis, and death mostly occurred within the first 24 hours. Rose et al. [1] reported that mortality was $28 \%$ among patients who had right heart thrombosis, and mortality was $100 \%$ among patients who did not undergo any treatment. Hence, when right atrium thrombus is found, rapid diagnosis and appropriate treatment should be done. Moreover, the treatment should not be postponed due to merely a morphological diagnosis.

Treatment of right atrium thrombus includes anticoagulant therapy with heparin, intravenous injection of thrombolytic 
agents, or surgical thromboembolectomy [3,11]. Thrombolytic therapy has a lower mortality than that of anticoagulant therapy and surgical treatment. Rose et al. [1] reported that the mortality for right heart thrombosis was $28.6 \%$ in anticoagulant therapy, $23.8 \%$ in surgical treatment, and $11.3 \%$ in thrombolytic therapy. Chartier et al. [9] reported that mortality in right atrium thrombosis was $47.1 \%$ in surgical treatment, $62.5 \%$ in anticoagulant therapy, and $22.2 \%$ in thrombolytic therapy. Thromboembolectomy intrinsically has potential complications including temporal delay, general anesthesia, cardiopulmonary bypass, and the lack of ability to eliminate a pulmonary thrombus existing beyond the central pulmonary artery [1]. However, we chose surgical elimination because there was a high possibility of hemorrhage during thrombolytic therapy since the patient was undergoing gynecological surgery and the thrombus in the right atrium was large.

The right atrium thrombus was spontaneously resolved during preparing for the thromboembolectomy. In spite of the continued transesophageal echocardiography, we were not able to discover the process in which the thrombus was spontaneously resolved. Only a few small thrombi were eliminated in the right pulmonary artery. We presume that the thrombus that had been in the right atrium entered into pulmonary circulation and then was resolved spontaneously.

In conclusion, we report a rare case in which a right atrial thrombus with unstable hemodynamic changes during laparoscopic assisted vaginal hysterectomy was detected by transesophageal echocardiography and resolved spontaneously during thromboembolectomy.

\section{References}

1. Rose PS, Punjabi NM, Pearse DB. Treatment of right heart thromboemboli. Chest 2002; 121: 806-14.
2. Schwartzbard AZ, Tunick PA, Rosenzweig BP, Kronzon I. The role of transesophageal echocardiography in the diagnosis and treatment of right atrial thrombi. J Am Soc Echocardiogr 1999; 12: 64-9.

3. Torbicki A, Perrier A, Konstantindes S, Agnelli G, Galie N, Pruszczyk $\mathrm{P}$, et al. Guidelines on the diagnosis and management of acute pulmonary embolism: the Task Force for the Diagnosis and Management of Acute Pulmonary Embolism of the European Society of Cardiology (ESC). Eur Heart J 2008; 29: 2276-315.

4. Obeid AI, al Mudamgha A, Smulyan H. Diagnosis of right atrial mass lesions by transesophageal and transthoracic echocardiography. Chest 1993; 103: 1447-51.

5. Pruszczyk P, Torbicki A, Pacho R, Chlebus M, Kuch-Wocial A, Pruszynski B, et al. Noninvasive diagnosis of suspected severe pulmonary embolism: transesophageal echocardiography vs spiral CT. Chest 1997; 112: 722-8.

6. Farfel Z, Shechter M, Vered Z, Rath S, Goor D, Gafni J. Review of echocardiographically diagnosed right heart entrapment of pulmonary emboli-in-transit with emphasis on management. Am Heart J 1987; 113: 171-8.

7. The European Cooperative Study on the clinical significance of right heart thrombi. European Working Group on Echocardiography. Eur Heart J 1989; 10: 1046-59.

8. Sung TY, Kim SH, Kim DK, Yoon TG, Kim TY, Lim JA, et al. Abrupt formation and spontaneous resolution of a right atrial thrombus detected by intraoperative transesophageal echocardiography during replacement of an abdominal aortic aneurysm. J Anesth 2010; 243: 456-59.

9. Chartier L, Bera J, Delomez M, Asseman P, Beregi JP, Bauchart JJ, et al. Free-floating thrombi in the right heart: diagnosis, management, and prognostic indexes in 38 consecutive patients. Circulation 1999; 99: 2779-83.

10. Chapoutot L, Nazeyrollas P, Metz D, Maes D, Maillier B, Jennesseaux C, et al. Floating right heart thrombi and pulmonary embolism: diagnosis, outcome and therapeutic management. Cardiology 1996; 87: 169-74.

11. Kearon C, Ginsberg JS, Douketis J, Trupie AG, Bates SM, Lee AY, et al. An evaluation of D-dimer in the diagnosis of pulmonary embolism: a randomized trial. Ann Intern Med 2006; 144: 812-21. 